\title{
Performance Enhancement of Multi-Band OFDM using Spectrum Equalizer
}

\author{
Sang-Hun Yoon, Jun-Mo Jung, Member, KIMICS
}

\begin{abstract}
In this paper, the equalization for frequency slope of path loss in Multi-Band(MB) OFDM UWB is proposed. The path loss of a signal is proportionate to the square of the signal's frequency. So, the received signal amplitudes of OFDM subcarrier can be different up to $3 \mathrm{~dB}$ when MB-OFDM occupies bandwidth over $1.5 \mathrm{GHz}$. The differences of subcarrier-amplitudes make an effective of 0.3 bit reduction of soft decision bits of viterbi decoder, and when the effective of 0.3 bit reduction can cause $0.5 \mathrm{~dB}$ SNR degradation. This paper proposes two modem architectures which compensate for the degraded subcarrier by multiplying the reciprocal of degraded values in analog or digital domain. It is shown that, for the proposed architecture applied to MB-OFDM UWB, the performance improvements up to $0.5 \mathrm{~dB}$ can be obtained over the conventional uncompensated receiver architecture.
\end{abstract}

Index Terms - Equalizer, Path loss, UWB, Viterbi Decoder

\section{INTRODUCTION}

The UWB suffers heavy band degradation, because the path loss of a signal power is proportionate to the square of the signal's frequency as in (1).

$$
\begin{aligned}
P L & =\left(\frac{4 \pi d}{\lambda}\right)^{2}=\left(\frac{4 \pi d}{c / f}\right)^{2}=\left(\frac{4 \pi d f}{c}\right)^{2} \\
& =\left(\frac{4 \pi d}{c}\right)^{2} \times f^{2}
\end{aligned}
$$

In (1), $d$ is a distance of between transmit and receive antenna, $\lambda$ is wavelength of used signal, $c$ is the speed of light, and $f$ is the frequency of the signal.

So, there can be existing slightly degraded and heavily diminished frequency parts. Because OFDM system carries information on frequency subcarrier, frequency

\footnotetext{
Manuscript received October 5, 2010; revised October 22, 2010; accepted November 2, 2010

Sang-Hun Yoon is with Sensor Interface Research Team, ETRI, Daejeon, Korea (Email: shyoon11@etri.re.kr), Jun-Mo Jung(Corresponding Author) is with the Department of Electronics Engineering, Kunsan National University, Kunsan, Korea(Email: jmjung@kunsan.ac.kr)
}

degradation means reduced input signal level of viterbi decoder. When a viterbi decoder received highly defected subcarrier as an input, the decoder can't use any information in MSB part of soft decision bits of subcarrier. This makes the viterbi decoder's gain reduced and the reduced decoder's gain increases bit error probability of the system.

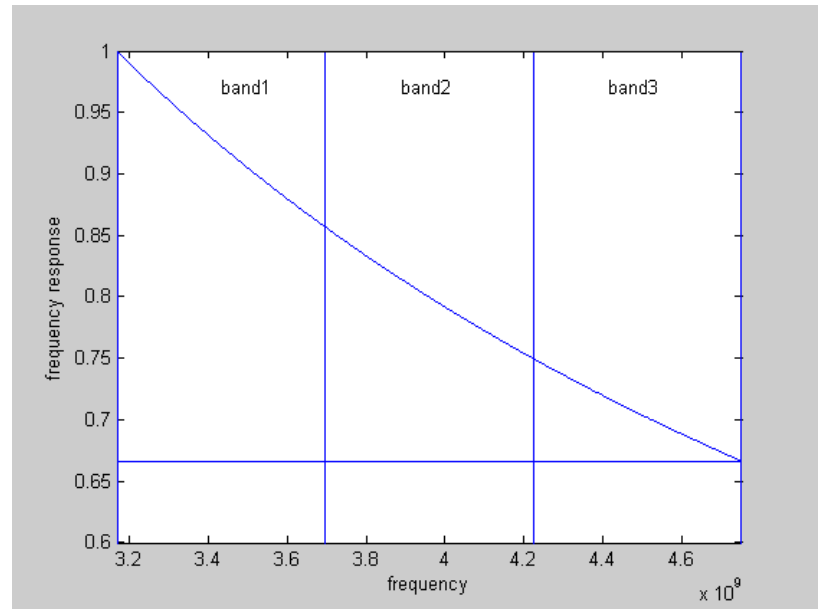

Fig. 1. Band degradation according to the path loss

Fig. 1 shows band frequency response according to the path loss on mandatory channel of MB-OFDM UWB, $3.168 \sim 4.762 \mathrm{GHz}$ as in [2].

This paper proposes an analog and a digital compensators to reduce the effect of soft decision bit reduction.

\section{PROPOSED COMPENSATOR}

\section{A. Band Degradation Effects}

Received signals, suffered for band degradation as in fig. 1, have smaller average signal level than that of original one. In (2) and (3), the average subcarrier signal level normalized by the best one is described. $X(w)$ is received signal level, and $P(w)$ is received signal power level. And, $f_{\min }, f_{\max }$ are minimum and maximum frequency in band, respectively. 


$$
\begin{aligned}
E\left[\frac{|P(w)|}{\left|P\left(f_{\min }\right)\right|}\right] & =\frac{f_{\min }^{2}}{f_{\text {max }}-f_{\min }} \int_{f_{\min }}^{f_{\max }} \frac{1}{f^{2}} d f \\
& =\frac{f_{\min }^{2}}{f_{\max }-f_{\min }}\left(\frac{1}{f_{\min }}-\frac{1}{f_{\max }}\right) \\
& =\frac{f_{\min }}{f_{\max }}
\end{aligned}
$$

$$
\begin{aligned}
E\left[\frac{|X(w)|}{\left|X\left(f_{\min }\right)\right|}\right] & =\frac{f_{\min }}{f_{\text {max }}-f_{\min }} \int_{f_{\min }}^{f_{\max }} \frac{1}{f} d f \\
& =\frac{f_{\min }}{f_{\text {max }}-f_{\min }}\left(\ln f_{\text {max }}-\ln f_{\min }\right) \\
& =\frac{f_{\min }}{f_{\text {max }}-f_{\min }} \ln \left(\frac{f_{\text {max }}}{f_{\min }}\right)
\end{aligned}
$$

In case of MB-OFDM UWB mandatory channel, the average signal power and the voltage level are reduced to $67 \%$ and $81 \%$, respectively. If N-bit soft decision is used, the average used bits in soft decision can be described as (4).

$$
\text { AveUsedSoftBits }=\log _{2}\left(0.81 \cdot 2^{N}\right)=N-0.30
$$

When 3 bit soft decision is used in a viterbi decoder, the effective soft decision bits are reduced to 2.7 bits, so that the reduction of effective soft decision bits can make $0.5 \sim 1 \mathrm{~dB}$ performance degradation.

\section{B. Analog Equalizer}

Path loss is a function of the frequency and the distance as in (1). Because the degradation according to the distance is adapted to all bands of frequencies, signals can be controlled through automatic gain control (AGC). Additionally, $f$ is the frequency of bands that we have already known.

If the received signal is equalized with a filter whose frequency response is proportionate to $f$, each frequency component of received signal can be even. Because three subbands hop frequency periodically and the hopped frequency signals are demodulated in receiver, RF equalizer is added to between LNA, which outputs all bands of frequencies, and Demodulator, which down converts RF signal into baseband signal. Because the input signals of equalizer contain AWGN as well as original signals, the equalizing of all bands can not get SNR improvement. But we can make the input signal levels of viterbi decoder even by compensation, and the even input level can minimize the bits loss of effective soft decision. Fig. 2 describes a structure of the MBOFDM UWB modem containing an analog equalizer.

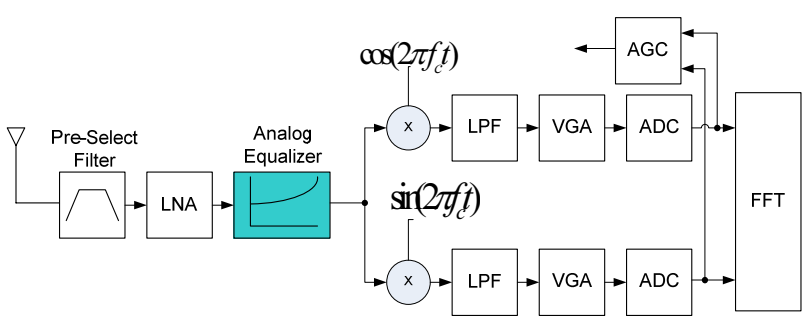

Fig. 2. UWB block diagram with Analog Equalizer

\section{Digital Compensator}

The effects of band degradation can also be compensated in baseband. Because FFT module exists already in baseband of OFDM system, compensation can be made by multiplying path loss to frequency domain signals after FFT operation. There is not only AWGN but also quantization noise in digital compensator input, so that the amplified quantization noise makes system performance worse than that of analog one. But quantization noise can be ignored when SNR is moderately small. In case of $(\mathrm{B}+1)$ bit resolution analogto-digital converter, the quantization noise of NSR is as in (5)[3].

$$
N S R=-6.02 B-10.8+20 \log \left(\frac{X_{m}}{\sigma_{x}}\right)
$$

In (5), $X_{m}$ is the full-scale amplitude of ADC and $\sigma_{x}$ is the root mean square(RMS) value of the signal amplitude. In case of $0 \mathrm{dBw}$ signal power and 5 bit $\mathrm{ADC}$, noise power is less than $-24 \mathrm{dBw}$. Fig. 3 shows modem architecture with digital compensator.

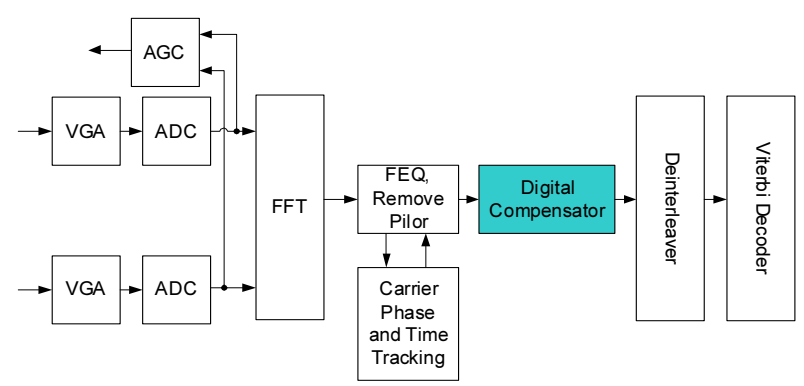

Fig. 3. UWB Modem Block Diagram with Digital Compensator

\section{SIMULATION RESULTS}

Proposed architecture is applied to MB-OFDM UWB which has been proposed as a standard of IEEE 802.15.3a [4]. Simulation is performed in $480 \mathrm{Mbps}$ mode using 3 bit soft decision viterbi decoder. Simulation environments and assumptions are listed in table I. 
TABLE I

SIMULATION ENVIRONMENTS AND ASSUMPTIONS

\begin{tabular}{|c|c|}
\hline \multicolumn{2}{|c|}{ Environments } \\
\hline System version & v.0891 \\
\hline ADC bits & 5 \\
\hline ADC Dynamic range & 480Mbps \\
\hline Data rate & Viterbi 3bit soft decision \\
\hline Channel decoder & 200 \\
\hline Packet length & \\
\hline Simulated packets & $\propto \mathrm{f} 2$ \\
\hline \multicolumn{2}{|c|}{ Assumption } \\
\hline Path Loss & Perfect \\
\hline Synch. \& Channel Eq. & $\mathrm{X}$ \\
\hline multipath &
\end{tabular}

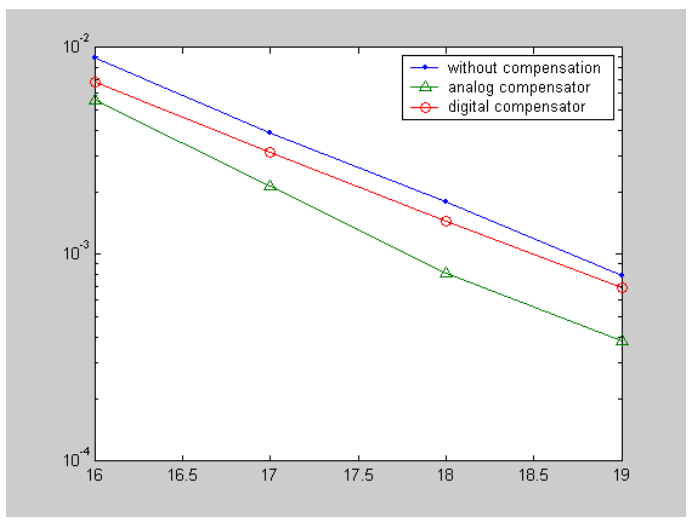

Fig. 4. BER performance of compensator

Fig. 4. shows bit error rate (BER) curve of simulation results. And as a result, analog and digital compensator can improve performance about $0.5 \sim 1 \mathrm{~dB}$.

\section{CONCLUSIONS}

This paper proposed a channel compensator which can maximize viterbi decoder gain by the compensation of degraded subcarrier. As the simulation results, the proposed analog compensator can make 0.5 1dB SNR improvements at $10^{-3}$ BER. Digital compensator can be used when the quantization noise is much smaller than AWGN. 5bit ADC is sufficient for $480 \mathrm{Mbps}$ mode of MB-OFDM. The SNR gain can be used to extend communication distance or to improve BER performance.

\section{REFERENCES}

[1] T.S.Rappaport, Wireless Communications, Upper Saddle River, NJ:Prentice Hall, 2002

[2] A.Batra, J.Balakrishman, A.Dabakand et al., "Multi-band OFDM Physical Layer Proposal for IEEE 802.15 Task Group 3a", IEEE P802.15-03/268r3, March 2004.

[3] A.V.Oppenheim, R.W.Schafer, Discrete-Time Signal Processing , Eaglewood Cliffs, NJ: Prentice Hall, 1989.

[4] J.R.Foerster, et al. "Channel Modeling Subcommitee Report Final," IEEE P802.1.5-02/490r1-SG3a, Feb. 2003.

[5] http://www.ieee802.org/15/pub/TG3a
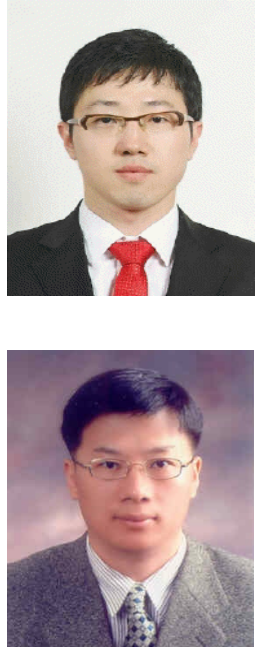

Sang-Hun Yoon received the $\mathrm{Ph}$. Degree from Hanyang University in 2008, and is currently work for Electronics and Telecommunications Research Institute in Daejeon, Korea, as a senior member of engineering staff since 2009. His research areas include ASIC design, Digital Signal Processing, Wireless Communication System Design, and Image Processing.

Jun-Mo Jung received the $\mathrm{Ph}$. Degree from Hanyang University in 2004, and is currently a professor at Dept. of Electronic Engineering at Kunsan National University, worked as a visiting Professor at Queensland Micro technology Facility at Griffith University in Australia in 2009 2010. His research areas include ASIC design, Digital Signal Processing and $\mathrm{NoC}$ test. 